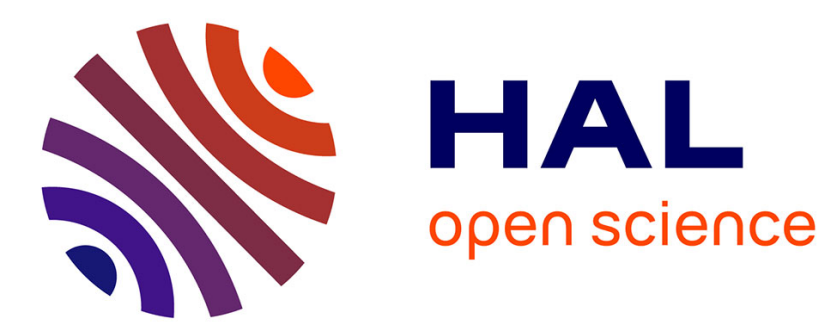

\title{
La confiance dans les relations interentreprises. Une revue des recherches quantitatives
}

Carole Donada, Gwenaëlle Nogatchewsky

\section{To cite this version:}

Carole Donada, Gwenaëlle Nogatchewsky. La confiance dans les relations interentreprises. Une revue des recherches quantitatives. Revue Française de Gestion, 2007, 33 (175), pp.111-124. halshs00340595

\section{HAL Id: halshs-00340595 \\ https://shs.hal.science/halshs-00340595}

Submitted on 8 Jul 2010

HAL is a multi-disciplinary open access archive for the deposit and dissemination of scientific research documents, whether they are published or not. The documents may come from teaching and research institutions in France or abroad, or from public or private research centers.
L'archive ouverte pluridisciplinaire HAL, est destinée au dépôt et à la diffusion de documents scientifiques de niveau recherche, publiés ou non, émanant des établissements d'enseignement et de recherche français ou étrangers, des laboratoires publics ou privés. 


\title{
La confiance dans les relations interentreprises : revue des recherches quantitatives
}

\author{
Carole DONADA* \\ Professeur Associé - ESSEC \\ BP 105 - 95021 Cergy Pontoise Cedex \\ donada@essec.fr \\ 01.34.43.30.68 \\ Gwenaëlle NOGATCHEWSKY** \\ Maître de Conférences - DRM-CREFIGE - CNRS UMR 7088 - \\ Université Paris Dauphine \\ Place du Maréchal de Lattre de Tassigny - 75116 Paris \\ nogatchewsky@yahoo.fr \\ 01.40.86.06.22
}

*Carole Donada est professeur à l'ESSEC. Ses recherches portent sur les stratégies d'alliances et les partenariats verticaux.

**Gwénaëlle Nogatchewsky est maître de conférences à l'Université Paris Dauphine et membre de DRM-CREFIGE. Ses recherches portent sur le contrôle dans les relations client-fournisseur. 


\title{
La confiance dans les relations interentreprises : revue des recherches quantitatives
}

\begin{abstract}
Cet article présente les principaux résultats des recherches quantitatives sur la confiance dans les relations interentreprises. Les auteurs distinguent les études réalisées selon leur objet de recherche (analyse des antécédents ou des conséquences de la confiance) et leur niveau d'analyse (le partenaire, la dyade, l'échange). La synthèse de ces études met en évidence le rôle central, positif et évolutif de la confiance dans les relations client-fournisseur.
\end{abstract}

This article presents the main results of quantitative studies about trust in interfirm relationships. The authors distinguish the studies on their research object (analysis of the antecedents and consequences of trust) and on the analysis level (the partner, the dyad, the exchange). The synthesis of these studies highlights the central, positive and evolutive role of trust in buyer-supplier relationships.

Théoriciens et praticiens ne cessent de débattre sur le rôle de la confiance dans les relations interentreprises. Pour certains, la confiance n'existe pas dans les affaires: "En tant qu'acheteur, la confiance, je ne sais pas ce que c'est. Tout est écrit dans mon cahier des charges.». Cette réflexion d'un professionnel fait écho aux propos de l'économiste Williamson (1993) pour qui les relations d'affaires résultent uniquement d'un calcul coût-bénéfice; la « vraie » confiance implique une dimension personnelle non rationnelle qui ne peut exister que dans la sphère privée. Pour d'autres au contraire, la confiance est la clé et le ciment des relations interentreprises : «Si on ne se faisait pas confiance, on n'y arriverait jamais. Plus on travaille ensemble, plus on se fait confiance et mieux on s'adapte aux circonstances.» ${ }^{1}$ Cette conception des relations basées sur la confiance est celle des théoriciens de l'approche relationnelle des échanges (Dwyer et al., 1987). Dans cette approche, les relations reposent sur une responsabilité partagée qui s'inscrit dans un climat de confiance.

Ces différentes conceptions du rôle de la confiance dans les relations interentreprises ont donné lieu à une multitude de travaux de recherche sur le sujet. En particulier, les travaux empiriques quantitatifs examinant les antécédents et les conséquences de la confiance sont riches d'enseignement. L'objectif de cet article est de présenter une synthèse des travaux statistiques considérés comme étant les plus représentatifs de la diversité des contributions 2 .

Au-delà de ses apports directs pour les chercheurs, cette synthèse peut offrir aux praticiens des résultats concrets et validés sur le rôle de la confiance dans leurs relations d'affaires. Les implications managériales de

\footnotetext{
${ }^{1}$ Dixit un acheteur d'un constructeur automobile.

2 Ces travaux ont été publiés dans des revues académiques internationales au cours des 15 dernières années. Les 37 articles retenus valident statistiquement des modèles dans lesquelles la confiance est soit une variable explicative ou une variable à expliquer. Seules les relations statistiques validées sont examinées.
}

ce travail seront développées en conclusion.

L'article est structuré en trois parties. La première partie rappelle brièvement comment les chercheurs définissent la confiance dans leur modèle théorique. Dans la seconde partie, un cadre méthodologique est proposé afin d'aider le lecteur à se repérer dans le grand nombre de travaux déjà réalisés. Ce cadre est présenté sous forme d'une figure (figure 1); il distingue les travaux selon leur objet de recherche (analyse des antécédents ou des conséquences de la confiance) et leur niveau d'analyse (sur le partenaire, la dyade, l'échange). La troisième partie de l'article reprend les principaux éléments que nous enseignent ces travaux de recherche

\section{I.- DE QUELLE CONFIANCE PARLENT LES CHERCHEURS ?}

Parmi les nombreuses définitions, deux conceptions sont souvent retenues.

La première considère que la confiance est une anticipation sur le fait que les attentes des partenaires ne seront pas déçues (Nooteboom et al., 1997 ; Zucker, 1986). C'est une probabilité que l'un n'abusera pas de l'autre et qu'il entreprendra les actions désirables pour la relation. Dès lors, tout ce qui contribue à restreindre les comportements opportunistes des partenaires (e.g. les contrats, les audits, les promesses de récompenses futures) est source de confiance.

La seconde conception suggère que la confiance est une croyance vis-à-vis d'autrui (Bidault et Jarillo, 1995 ; Guibert, 1999 ; Puthod, 1995 ; Sako, 1992). Avoir confiance en quelqu'un, c'est croire qu'il peut et veut agir de façon positive. Cette croyance repose sur deux arguments : 1) un argument technique qui lie la confiance aux compétences des partenaires (i.e. croyance dans les capacités de l'autre à réaliser la tâche - crédibilité du partenaire) ; 2) un argument moral qui met l'accent sur l'honnêteté et la bonne volonté des partenaires (i.e., croyance dans l'engagement moral de l'autre à agir de façon positive en cas d'imprévu bienveillance du partenaire). Dès lors, la confiance se 
fonde sur la réputation des partenaires et sur leurs expériences passées. Elle se construit par le développement de liens socio-psychologiques et par des processus d'apprentissage. C'est généralement à cette seconde conception que les chercheurs en management se réfèrent.

Certains différencient dans leurs travaux la confiance interpersonnelle de la confiance interorganisationnelle. La confiance interpersonnelle concerne les interlocuteurs intuitu personae des échanges. La confiance interorganisationnelle désigne une orientation collective de confiance entre les individus des firmes partenaires (Zaheer et al., 1998). Les processus qui permettent de développer cette orientation collective ne sont pas toutefois pas très explicites (Guibert, 1999).

\section{II.- DIFFERENTS NIVEAUX D'ANALYSE POUR ETUDIER LA CONFIANCE}

La figure 1 met en évidence les objets d'étude des recherches sur la confiance interentreprises. Elle distingue les antécédents et les conséquences de la confiance interentreprises selon trois niveaux d'analyse : celui des partenaires, celui de la dyade et celui de l'échange.

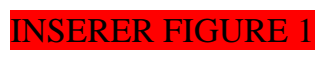

\section{Trois niveaux d'antécédents}

Au premier niveau, les antécédents de la confiance sont directement liés aux partenaires (i.e. l'entreprise dans son ensemble, voire une filiale ou un département). Ils ont trait aux caractéristiques intrinsèques des partenaires (taille, expertise, prédispositions à la confiance) au sein de leur environnement (réputation, performance, orientation marché).

Le deuxième niveau est celui de la dyade (i.e. de la relation entre deux entreprises). Les antécédents de la confiance sont ici liés aux caractéristiques de la dyade c'est-à-dire à l'interdépendance des partenaires, à l'équilibre de leurs pouvoirs, à leurs investissements spécifiques, au partage de leurs valeurs, à la convergence de leurs buts et à l'âge de leur relation.

Le troisième niveau est celui de l'échange proprement dit (i.e. de la relation entre les personnes responsables de l'échange). Les antécédents de la confiance portent cette fois sur les caractéristiques des échanges entres les partenaires : le déroulement de la coopération (comportements coopératifs, opportunisme, conflit), la qualité de leurs interactions (communication, fréquence des contacts) et les affinités entre les individus impliqués dans l'échange (sympathie, similitude, satisfaction dans l'échange).

\section{Trois niveaux de conséquences}

Par souci de symétrie et conformément à la représentation de la figure 1 , les conséquences de la confiance sont présentées du niveau 3 (niveau de l'échange) au niveau 1 (niveau des partenaires).

Les conséquences de troisième niveau portent essentiellement sur les modalités de gouvernance des échanges. Cette gouvernance peut être relationnelle ou hiérarchique. La gouvernance relationnelle se traduit par le respect des normes relationnelles (équité, partage de l'information, résolution harmonieuse du conflit), par des actions coopératives (action et planning joints, absence d'opportunisme) ou encore par des efforts de coordination et d'implication des partenaires. La gouvernance hiérarchique se traduit par des mécanismes d'influence et de contrôle (protection, sanction, coercition).

Les conséquences de deuxième niveau concernent la dyade et, plus particulièrement, le futur de la dyade. La confiance peut avoir des implications en termes d'engagement, de continuité ou d'orientation de long terme.

Les conséquences de premier niveau portent sur les partenaires et, plus particulièrement, sur la performance de la relation interentreprises pour les partenaires. La confiance peut avoir des implications en termes d'économies sur les coûts de transaction ou sur les coûts de négociation, de croissance des ventes ou encore de réduction de l'incertitude.

\section{III.- QUELS SONT LES RESULTATS DES RECHERCHES QUANTITATIVES ?}

L'analyse des recherches empiriques quantitatives conduites ces quinze dernières années met en lumière les antécédents et les conséquences les plus significatifs de la confiance interentreprises. Les tableaux 1 et 2 synthétisent les résultats des hypothèses testées et validées par les chercheurs.

\section{Les antécédents les plus significatifs}

INSERER TABLEAU 1

Parmi les sept antécédents du premier niveau reflétant les caractéristiques intrinsèques des partenaires, les plus étudiés sont: (1) les effets de la performance, (2) les effets de la réputation et (3) les effets de l'expertise.

1. La notion de performance revêt des aspects divers selon les études. Alors que Bharadwaj et Matsuno (2006), Chryssochoidis et Theoharakis (2004), Gounaris (2005) retiennent des indicateurs de performance opérationnelle (en particulier la qualité), Anderson et Narus (1990) se focalisent sur les 
indicateurs de performance concurrentielle (en particulier la compétitivité prix). Mais, quelque soient les indicateurs choisis, tous ont une influence positive sur la confiance.

2. La notion de réputation comme antécédent majeur de la confiance est à expliciter. Pour Doney et Cannon (1997), la réputation est la reconnaissance de l'honnêteté et de l'empathie de l'entreprise au sein de son environnement. Elle concerne davantage les prédispositions relationnelles de la firme que les compétences (liées à la notion d'expertise). Les résultats des études montrent sans surprise qu'une réputation positive favorise la confiance (principalement étudiée d'un point de vue inter-organisationnel) tandis qu'une réputation négative la dessert.

3. La notion d'expertise fait référence au savoir et savoir-faire du partenaire. L'expertise participe à la fois au développement de la confiance inter-personnelle et inter-organisationnelle.

Les travaux les plus récents sur les antécédents de niveau 2 distinguent la confiance interorganisationnelle (nommée parfois crédibilité) et la confiance inter-personnelle (nommée parfois bienveillance) pour montrer en fait que les caractéristiques de la dyade ont une influence sur les deux types de confiance. Ces caractéristiques les plus significatives sont liées (1) aux relations de dépendance et de pouvoir entre les entreprises, (2) à la satisfaction sur les interactions passées et (3) aux similitudes des entreprises. Dans une méta-analyse des antécédents de la confiance, Palmatier et al. (2006) montrent que les similitudes influencent davantage la confiance que la satisfaction des partenaires sur leurs interactions passées ou leur niveau de dépendance.

1. La dépendance de l'un des partenaires (qui peut se traduire par des investissements spécifiques engagés par l'un des partenaires) ou l'interdépendance entre les partenaires favorisent la confiance.

2. La satisfaction sur les interactions passées favorise la confiance. La méta-analyse de Geykens et al. (1999) insiste sur le rôle majeur de la satisfaction des partenaires dans les relations interentreprises.

3. Les Similitudes interentreprises s'expriment dans des valeurs communes, des buts partagés ou des liens sociaux et culturels. Elles sont fortement créatrices de confiance.

Parmi les antécédents ayant trait aux caractéristiques des échanges, les chercheurs distinguent nettement les antécédents liés: (1) aux comportements dysfonctionnels, (2) aux comportements relationnels et (3) aux expériences affectives des individus dans l'échange.

1. Les comportements dysfonctionnels se traduisent par des conflits et des comportements opportunistes. Sans surprise, les chercheurs confirment leur influence négative sur la confiance. La méta-analyse de Palmatier et al. (2006) révèle que l'effet négatif $\mathrm{du}$ conflit sur la confiance est beaucoup plus fort que l'effet positif d'une stratégie relationnelle qui la favorise.

2. Les comportements relationnels sont fondés sur la communication, le partage d'information, l'échange relationnel, la flexibilité, l'assistance, l'attente de continuité. Ils constituent les normes relationnelles décrites par Macneil (1980). Les liens positifs entre les comportements relationnels et la confiance ont d'abord été mis en valeur par Morgan et Hunt (1994). Depuis, leur modèle sert de fondement à la plupart des travaux s'inscrivant dans l'approche relationnelle.

3. Les expériences affectives expriment la sympathie et la proximité culturelle entre les individus. Les travaux montrent qu'elles ont une influence positive sur la confiance interpersonnelle, mais pas nécessairement sur la confiance inter-organisationnelle. Le passage du niveau interpersonnel au niveau interorganisationnel ne semble pas aisé. Seul Zaheer et al. (1998) montrent l'influence réciproque positive des deux types de confiance.

\section{Les conséquences les plus significatives}

\section{INSERER TABLEAU 2}

L'analyse des résultats des recherches montrera la confiance a des conséquences aussi bien sur la gouvernance relationnelle que sur la gouvernance hiérarchique.

Les conséquences de premier niveau ont trait aux effets de la confiance sur la performance des partenaires. Les études valident toutes l'influence positive de la confiance sur la performance des partenaires. La portée managériale de ce résultat est importante si l'on considère la performance comme l'une des premières finalités de l'entreprise. Et pourtant, le lien confiance-performance n'est intuitivement pas évident. Une relation est économiquement performante lorsque l'une ou les deux parties en tirent un avantage financier ou concurrentiel évalué de manière objective (augmentation des ventes, des profits, de l'avantage compétitif, réduction des coûts, etc.). Or, la confiance en tant que « croyance visà-vis d'autrui » comporte une dimension largement subjective liée à l'engagement moral de l'autre, à sa bienveillance. Dès lors, parvenir à démontrer le lien entre une dimension subjective de croyance et une dimension objective de performance constitue un apport majeur de la recherche sur le management des relations interentreprises.

Les conséquences de deuxième niveau portent sur le 
futur de la dyade. Les études valident l'influence positive de la confiance sur la volonté d'engagement des partenaires, l'étendue et la continuité3 de leur relation. Le lien confiance-engagement est le plus traité par les chercheurs depuis la théorie confianceengagement proposée par Morgan et Hunt en 1994. Il est aussi reconnu que la confiance se développe dans le temps entre les partenaires (Dwyer et al., 1987) selon un cercle vertueux; la confiance s'approfondit au fur et à mesure que la relation se développe. Sa nature devant sans doute évoluer, certains auteurs appellent à une analyse plus fine et longitudinale des types et des niveaux de confiance aux différents stades de la relation.

Les chercheurs ont exploré les conséquences de la confiance sur les modalités de gouvernance des échanges: (1) la gouvernance relationnelle et (2) la gouvernance hiérarchique.

1. Les résultats empiriques confirment très largement l'influence positive de la confiance sur la gouvernance relationnelle. Ce type de gouvernance se traduit par davantage de coopération, d'action jointe, de coordination, d'implication des partenaires, d'acceptation de normes relationnelles (partage d'informations, résolution harmonieuse des conflits, communication). Ces observations sont une nouvelle fois en ligne avec les propositions des théoriciens de l'échange relationnel (Dwyer et al., 1987 ; Macneil, 1980).

2. Quelques études établissent le lien entre confiance et gouvernance hiérarchique. Leurs résultats montrent que la confiance a une influence négative sur le contrôle, sur la coercition et sur les besoins de protection des partenaires. Le lien confiance-contrôle reste toutefois discuté par les chercheurs. Pour la plupart, la confiance est une alternative au contrôle. Dekker (2004), Nooteboom et al. (1997) ou Woolthuis et al. (2005) indiquent par exemple que le contrôle détruit la confiance, au moins à partir d'un certain niveau de proximité relationnelle, car il entraîne la suspicion. Gosse et al. (2002) suggèrent que la substitution du contrôle par la confiance au contrôle formel s'explique lorsque le client ne sait évaluer la prestation de son fournisseur. D'autres chercheurs considèrent au contraire que la gouvernance hiérarchique (i.e. le contrôle formel) et la gouvernance relationnelle (i.e. le contrôle informel) sont complémentaires pour créer un «climat de confiance» entre les partenaires (Guibert et Dupuy, 1997). En effet, l'élaboration et la mise en œuvre de mécanismes de contrôle, qui nécessitent la multiplication des interactions entre les

3 Seuls Bharadwaj et Matsuno (2006) observent une influence négative de la confiance sur la continuité. Les auteurs, surpris de ce résultat inattendu, ne parviennent à l'expliquer. partenaires, ne sont efficaces que si elles s'appuient sur une confiance mutuelle. Réciproquement, la connaissance partagée, favorisée par la confiance, permet un raffinement des dispositifs de contrôle. Contrôle et confiance peuvent donc se développer ensemble de manière cohérente. Les résultats de l'étude de Dumoulin et al. (2000), qui rejettent l'hypothèse de la relation substitutive entre le contrat et la confiance, et de celle de Guibert et Dupuy (1997), qui n'identifient pas de lien entre la confiance et la formalisation contractuelle du contrôle, abondent dans ce sens.

Il apparaît enfin que les conséquences de la confiance sur les différents aspects du déroulement des relations interentreprises ne sont pas de même intensité. Dans leur méta-analyse, Palmatier et al. (2006) ainsi constatent que l'impact de la confiance est le plus fort sur la gouvernance relationnelle (en particulier en termes de coopération), puis sur le futur de la dyade et enfin sur la performance économique des partenaires.

\section{CONCLUSION}

Cette étude met en évidence le rôle central de la confiance dans les relations interentreprises ${ }^{4}$. Ce rôle est largement validé par les nombreuses études quantitatives publiées au cours de ces quinze dernières années. Deux enseignements managériaux majeurs méritent d'être soulignés.

D'abord, les implications positives de la confiance sur le développement des relations révèlent le rôle important qu'il est souhaitable d'accorder à la confiance dans les échanges. Elle apporte une plus grande performance économique et opérationnelle aux partenaires. Elle leur permet de se projeter ensemble dans le futur avec moins d'incertitude. Elle est aussi la garantie de déplacer une relation contractuelle contraignante, fondée sur une gouvernance hiérarchique, vers une coopération ouverte et flexible, fondée sur une gouvernance relationnelle plus adaptée à la réussite dans un contexte de plus en plus changeant.

En outre, cette étude insiste sur la nécessaire compréhension des mécanismes de construction de la confiance, ce qui est un enjeu crucial pour les praticiens. C'est le second enseignement majeur de cette synthèse. Pour susciter la confiance, les entreprises ont intérêt à soigner leur réputation, à valoriser leur expertise dans leur domaine et à afficher leurs performances. Elles doivent également être attentives aux caractéristiques de la dyade pouvant favoriser la confiance : la dépendance réciproque, les

4 Et tout particulièrement sur les relations clientfournisseur. 
bonnes expériences passées ainsi qu'une similitude, une certaine proximité dans les valeurs et les buts des deux entreprises. Mais c'est sans doute dans l'exercice même de la relation, dans la conduite des échanges, que la confiance peut le mieux se construire: le partage d'informations, la communication, la flexibilité et l'engagement sont autant d'éléments sur lesquels l'entreprise peut s'appuyer pour développer solidement la confiance de l'autre et donc favoriser une coopération fructueuse et durable avec lui.

Ces enseignements généraux peuvent être suivis d'effets concrets. Ainsi, certaines entreprises dédient des ressources spécifiques au développement des normes relationnelles telles que la flexibilité, la solidarité, l'échange d'information (en particulier les entreprises en situation de dépendance asymétrique visà-vis de leurs partenaires). D'autres développent une véritable stratégie de la confiance. C'est ce que révèle Neuville (1997) dans une recherche sur le partenariat dans l'industrie automobile. L'auteur montre comment des fournisseurs utilisent la confiance pour rassurer leur client dans un premier temps et mieux masquer, par la suite, leurs défaillances et gains de productivité. Cette étude rappelle que la confiance porte en elle-même les racines de l'opportunisme parce qu'elle engendre une baisse de la vigilance et du contrôle de celui qui fait confiance. Mais, si le stratège opportuniste est démasqué, la sanction est sévère. Les conséquences négatives dépassent le cadre de la dyade. Le comportement opportuniste détériore la réputation d'un partenaire au sein de son réseau d'affaires. Or, le BCG5 souligne l'importance de la réputation dans un monde où l'information circule vite et librement par une multitude de canaux. La réputation est un actif propre à chacun non reconnu par les conventions comptables. Elle fonde la confiance. De son côté, la confiance doit être considérée comme un actif spécifique à la relation qui ne doit pas être négligé. Lorsque la relation se termine, la confiance entre les partenaires cesse d'être effective dans les échanges, mais elle prend une autre forme. Elle vient alimenter la bonne réputation de l'entreprise au sein de son réseau d'affaires.

5 «De la réciprocité à la réputation», BCG Perspectives, 2006. 
Figure 1 : Les objets des études sur la confiance interentreprises

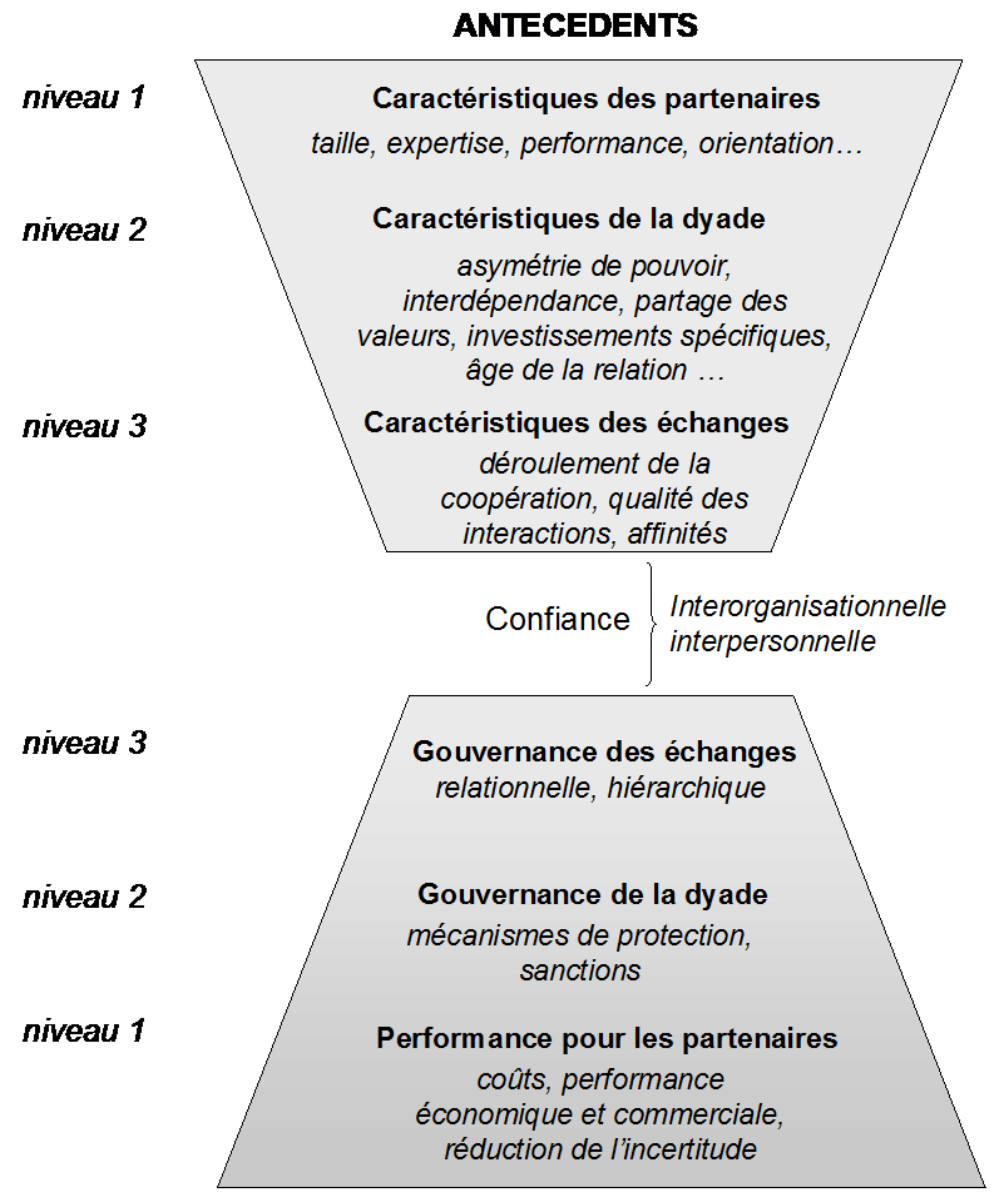

\section{CONSEQUENCES}


Tableau 1 : Résultats des recherches sur les antécédents de la confiance

\begin{tabular}{|c|c|c|}
\hline \multicolumn{3}{|c|}{ Niveau 1 : Caractéristiques des partenaires } \\
\hline Performance & $(+)$ & $\begin{array}{l}\text { Anderson et Narus (1990) ; Bharadwaj et Matsuno (2006); Chryssochoidis } \\
\text { et Theoharakis (2004); Gounaris (2005); Wiertz et al. (2004) }\end{array}$ \\
\hline Réputation & $(+)$ & $\begin{array}{l}\text { Anderson et Weitz (1989) ; Chu et Fang (2006) ; Doney et Cannon } \\
(1997) * \text {; Ganesan }(1994) \text {; Lee et Dawes }(2004) * \text { et ** ; Lui et al. (2006)* }\end{array}$ \\
\hline Expertise & $(+)$ & $\begin{array}{l}\text { Andaleeb }(1996) * * ; \text { Doney et Cannon (1997) ; Lee et Dawes }(2004) * \text { et } \\
* * ; \text { Selnes }(1998)\end{array}$ \\
\hline Prédisposition à la confiance & $(+)$ & Andaleeb (1996)**; Gao et al. (2005) \\
\hline Orientation marché & $(+)$ & Doney et Cannon (1997)* ; Siguaw et al. (1998) \\
\hline Taille & $(+)$ & Doney et Cannon $(1997)^{*}$ \\
\hline Engagement du partenaire & $(+)$ & Gao et al. (2005) ; Miyamoto et Rexha (2004) \\
\hline \multicolumn{3}{|c|}{ Niveau 2 : Caractéristiques de la dyade } \\
\hline Dépendance & $(+)$ & Gao et al. (2005) ; Kumar et al. (1995) \\
\hline Interdépendance & $(+)$ & Kumar et al. (1995) \\
\hline Asymétrie de pouvoir & $(-)$ & Anderson et Weitz (1989) \\
\hline $\begin{array}{l}\text { Satisfaction sur les } \\
\text { interactions passées }\end{array}$ & $(+)$ & $\begin{array}{l}\text { Ganesan (1994)* et **; Guibert (1999) ; Lui et al. (2006)** ; Miyamoto et } \\
\text { Rexha (2004) }\end{array}$ \\
\hline Age de la relation & $(+)$ & Anderson et Weitz (1989) ; Doney et Cannon (1997) ; Dyer et Chu (2000) \\
\hline Ressemb. interentreprises & $(+)$ & Lui et al. (2006)** \\
\hline Partage des valeurs & $(+)$ & Guibert (1999) ; Morgan et Hunt (1994) ; MacMillan et al. (2005) \\
\hline Convergence des buts & $(+)$ & Anderson et Weitz (1989) \\
\hline Liens sociaux et structurels & $(+)$ & Gounaris (2005) \\
\hline Investissements spécifiques & $(+)$ & Ganesan (1994)* et **; Lui et al. (2006)* \\
\hline
\end{tabular}

\section{Niveau 3 : Caractéristiques des échanges}

\begin{tabular}{lll}
\hline Opportunisme & $(-)$ & $\begin{array}{l}\text { Andaleeb (1996)**; Guibert (1999) ; MacMillan et al. (2005) ; Morgan et } \\
\text { Hunt (1994) }\end{array}$ \\
\hline Conflit & $(-)$ & Chu et Fang (2006) \\
\hline Satisfaction dans l'échange & $(+)$ & Chu et Fang (2006) ; Selnes (1998) \\
\hline Echange relationnel & $(+)$ & Aulakh et al. (1996) ; Siguaw et al. (2003) ; Wiertz et al. (2004) \\
\hline Communication & $(+)$ & $\begin{array}{l}\text { Anderson et Narus (1990) ; Anderson et Weitz (1989) ; Chu et Fang } \\
(2006) \text {; Doney et Cannon (1997)** ; Dyer et Chu (2000) ; Guibert (1999) ; }\end{array}$ \\
\hline Partage d'informations & $(+)$ & Aulakh et al. (1996) ; Doney et Cannon (1997) \\
\hline Continuité attendue & $(+)$ & Aulakh et al. (1996) ; Dyer et Chu (2000) \\
\hline Flexibilité & $(+)$ & Aulakh et al. (1996) \\
\hline Assistance & $(+)$ & Anderson et Weitz (1989) ; Dyer et Chu (2000) \\
\hline Engagement & $(+)$ & Brulhart (2002) ; Medlin et al. (2005) \\
\hline Equité & $(+)$ & Brulhart (2002) \\
\hline Proximité culturelle & $(+)$ & Anderson et Weitz (1989); Doney et Cannon (1997)** \\
\hline Sympathie & $(+)$ & Andaleeb (1996)** ; Doney et Cannon (1997)** \\
\hline
\end{tabular}

* confiance interorganisationnelle

** confiance interpersonnelle 
Tableau 2 : Résultats des recherches sur les conséquences de la confiance

\begin{tabular}{lll}
\hline Niveau 1 : Performance de la relation pour les partenaires \\
\hline$(+)$ & Performance & $\begin{array}{l}\text { Aulakh et al. (1996) ; Bulhart (2002); Corsten et Kumar (2005) ; Dyer } \\
\text { et Chu (2003); MacMillan et al. (2005); Medlin et al. (2005) ; Sako } \\
(1998) ; \text { Siguaw et al. (2003) }\end{array}$ \\
\hline$(-)$ & Coûts de transaction & Bharadwaj et Matsuno (2006); Dyer et Chu (2003) \\
\hline$(-)$ & Coûts de négociation & Zaheer et al. (1998)* \\
\hline$(-)$ & Incertitude & Gao et al. (2005); Morgan et Hunt (1994) \\
\hline $\begin{array}{l}\text { ni (+) } \\
\text { ni (-) }\end{array}$ & Avantage compétitif & Chryssochoidis et Theoharakis (2004) \\
\hline
\end{tabular}

Niveau 2 : Futur de la dyade

\begin{tabular}{|c|c|c|}
\hline$(+)$ & Engagement & $\begin{array}{l}\text { Abduhl-Muhmin }(2005)^{*} \text {; Chu et Fang (2006) ; Gounaris (2005); } \\
\text { Grayson et Ambler (1999); Guibert (1999); Lohtia et al. (2005); } \\
\text { Moorman et al. (1992); Morgan et Hunt (1994) }\end{array}$ \\
\hline$(+)$ & Etendue de la coopération & Selnes (1998) \\
\hline$(+)$ & Continuité & $\begin{array}{l}\text { Anderson et Weitz (1989) ; Doney et Cannon (1997)* et ** ; Ganesan } \\
(1994)^{*} \text {; Lee et Dawes (2004)* et **; Siguaw et al. (2003) }\end{array}$ \\
\hline$(-)$ & Continuité & Bharadwaj et Matsuno (2006) \\
\hline
\end{tabular}

\section{Niveau 3 : Gouvernance des échanges \\ Gouvernance relationnelle}

\begin{tabular}{lll}
\hline$(+)$ & Coopération & $\begin{array}{l}\text { Anderson et Narus (1990); Morgan et Hunt (1994); Siguaw et al. } \\
(2003) ; \text { Wiertz et al. (2004) }\end{array}$ \\
\hline$(+)$ & Action jointe & $\begin{array}{l}\text { Claro et al. (2003)* et **; Payan (2006)* et **; Sako (1998); Zaheer } \\
\text { et Venkatraman (1995) }\end{array}$ \\
\hline$(+)$ & Implication & Grayson et Ambler (1999); Moorman et al. (1992) \\
\hline$(+)$ & Qualité des interactions & Grayson et Ambler (1999); Moorman et al. (1992) \\
\hline$(-)$ & Opportunisme & Grayson et Ambler (1999); Smith et Barclay (1997) \\
\hline$(+)$ & Coordination & Jap (1999)** \\
\hline$(+)$ & Communication & Anderson et Weitz (1989); Smith et Barclay (1997) \\
\hline$(+)$ & Partage d'informations & Dyer et Chu (2003) \\
\hline$(+)$ & Résolution de conflit & Anderson et Narus (1990); Morgan et Hunt (1994) \\
\hline$(-)$ & Conflit & Zaheer et al. (1998)* et **
\end{tabular}

Gouvernance hiérarchique

\begin{tabular}{lll}
\hline$(-)$ & Contrôle & Andaleeb (1995); Guibert et Dupuy (1997) \\
\hline$(-)$ & Mécanismes de protection & Schumacher (2006) \\
\hline$(-)$ & Influence coercitive & Simpson et Mayo (1997) \\
\hline$*$ confiance interorganisationnelle & \\
$* *$ confiance interpersonnelle &
\end{tabular}




\section{BIBLIOGRAPHIE}

A.G. Abdul-Muhmin, «Instrumental and interpersonal determinants of relationship satisfaction and commitment in industrial markets ", Journal of Business Research, Vol. 58, ํㅜ 5, p. 619-628, 2005.

S.S. Andaleeb, «Dependence relations and the moderating role of trust: implications for behavioral intentions in marketing channels », International Journal of Research in Marketing, Vol. 12, $\mathrm{N}^{\circ}$ 2, p. 154-172, 1995.

S.S. Andaleeb, « Factors Influencing Customer Trust in Salespersons in a Developing Country », Journal of International Marketing, Vol. 4, № 4, p. 35-52, 1996.

E. Anderson, B. Weitz, « Determinants of continuity in conventional industrial channel dyads », Marketing Science, Vol. 8, ํ4, p. 310-323, 1989.

J.C. Anderson, J.A. Narus, «A Model of Distributor Firm and Manufacturing Firm Working Partnerships », Journal of Marketing, Vol. 54, $\mathrm{N}^{\circ} 1$, p. 42-58, 1990.

P.S. Aulakh, M. Kotabe, A. Sahay, «Trust and Performance in Cross-Border Partnerships : A Behavioral Approach », Journal of International Business Studies, Special Issue, p. 1105-1132, 1996.

N. Bharadwaj, K. Matsuno, «Investigating the antecedents and outcomes of customer firm transaction cost savings in a supply chain relationship », Journal of Business Research, Vol. 59, N 1, p. 62-72, 2006.

F. Bidault, J.C. Jarillo, «La confiance dans les transactions économiques », in F. Bidault, P.Y. Gomez et G. Marion (eds), Confiance, Entreprise et Société, Eska, Paris, p. 109-123, 1995.

F. Brulhart, «Le rôle de la confiance dans le succès des partenariats verticaux logistiques :le cas des coopérations entre industriels agro-alimentaires et prestataires logistiques », Finance Contrôle Stratégie, Vol. 5, N4, p. 51-77, 2002.

G. Chryssochoidis, V. Theoharakis, «Attainment of competitive advantage by the exporter-importer dyad: The role of export offering and import objectives », Journal of Business Research, Vol. 57, N ${ }^{\circ} 4$, p. 329337, 2004.

S-Y. Chu, W-C. Fang, «Exploring the relationships of trust and commitment in supply chain management », Journal of American Academy of Business, Vol. 9, $\mathrm{N}^{\circ}$ 1, p. 224-228, 2006.

D.P. Claro, G. Hagelaar, O. Omta, « The determinants of relational governance and performance: how to manage business relationships? », Industrial Marketing Management, Vol. 32, N 8, p. 703-176, 2003.

D. Corsten, N. Kumar, «Do suppliers benefit from collaborative relationships with large retailers? An empirical investigation of efficient consumer response adoption », Journal of Marketing, Vol. 69, N 3, p. 8094, 2005.
H.C. Dekker, « Control of inter-organizational relationships: evidence on appropriation concerns and coordination requirements», Accounting, Organizations \& Society, Vol. 29, $\mathrm{N}^{\circ}$ 1, p.27-50, 2004.

P.M. Doney, J.P. Cannon, «An examination of the nature of trust in buyer-seller relationships », Journal of Marketing, Vol. 61, $\mathrm{N}^{\circ}$ 2, p. 35-51, 1997.

F. R. Dwyer, P. H. Schurr, S. Oh, « Developing buyerseller relationships », Journal of Marketing, Vol. 51, $\mathrm{N}^{\circ} 2$, p. 11-27, 1987.

J.H. Dyer, W. Chu, «The Determinants of Trust in Supplier-automaker Relationships in the US, Japan and Korea ", Journal of International Business Studies, Vol. 31, $\mathrm{N}^{\circ}$ 2, p. 259-285, 2000.

J.H. Dyer, W. Chu, «The role of trustworthiness in reducing transaction costs and improving performance: empirical evidence from the United States, Japan, and Korea », Organization Science, Vol. 14, N¹, p. 57-68, 2003.

S. Ganesan, «Determinants of long-term orientation in buyer-seller relationships », Journal of Marketing, Vol. 58, $\mathrm{N}^{\circ} 2$, p. 1-19, 1994.

T. Gao, M. Joseph Sirgy, M.M. Bird, « Reducing buyer decision-making uncertainty in organizational purchaing: Can supplier trust, commitment, and dependence help?», Journal of Business Research, Vol. 58, N 4, p. 397-405, 2005.

I. Geykens, J.B. Steenkamp, N. Kumar, «Generalizations about trust in marketing channel relationships using meta-analysis », International Journal of Research in Marketing, Vol. $15 \mathrm{~N}^{\circ}$ 3, p.223248, 1998.

B. Gosse, C. Sargis-Roussel, P.A. Sprimont, «Les changements organisationnels liés aux stratégies d'externalisation:le cas d'une entreprise industrielle », Finance Contrôle Stratégie, Vol. 5, º1, p.101-128, 2002.

S.P. Gounaris, «Internal-market orientation and its measurement », Journal of Business Research, Vol. 59, $\mathrm{N}^{\circ}$ 4, p. 432-448, 2005.

K. Grayson, T. Ambler, «The dark side of long-term relationships in marketing services ", Journal of Marketing Research, Vol. 36, N 1, p. 132-141, 1999.

N. Guibert, « La confiance en marketing : fondements et applications », Recherche et Applications en Marketing, Vol 14, $\mathrm{N}^{\circ}$ 1, p. 1-19, 1999.

N. Guibert, Y. Dupuy, «La complémentarité entre contrôle «formel » et « contrôle informel »: le cas de la relation client-fournisseur », Comptabilité Contrôle Audit, tome 3, Vol. 1, p. 39-52, 1997.

S.D. Jap, «Pie-expansion efforts: collaboration processes in buyer-supplier relationships », Journal of Marketing Research, Vol. 36, № 4, p. 461-475, 1999.

N. Kumar, L.K. Scheer, J-B. E.M. Steenkamp, « The Effects of Perceived Interdependence on Dealer 
Attitudes », Journal of Marketing Research, Vol. 32, p. 348-356, August 1995.

D.Y. Lee, P.L. Dawes, «Guanxi, trust, and long-term orientation in Chinese business markets », Journal of International Marketing, Vol. 13, $\mathrm{N}^{\circ}$ 2, p. 28-56, 2005.

R. Lohtia, D.C. Bello, T. Yamada, D. Gilliland, «The role of commitment in foreign-Japanese relationships: mediating performance for foreign sellers in Japan», Journal of Business Research, Vol. 58, N 8, p.10091018, 2005.

S.S. Lui, H-Y. Ngo, A.H.Y. Hon, « Coercive strategy in interfirm cooperation : Mediating roles of interpersonal and interorganizational trust », Journal of Business Research, Vol. 59, N 4, p. 466-474, 2006.

K. MacMillan, K. Money, A. Money, S. Downing, «Relationship marketing in the non-profit sector: an extension and application of the commitment-trust theory », Journal of Business Research, Vol. 58, $\mathrm{N}^{\circ} 6$, p. 806-818, 2005.

C.J. Medlin, J-M. Aurifeille, P.G. Quester, «A collaborative interest model of relatinoal coordination and empirical results », Journal of Business Research, Vol. 58, $\mathrm{N}^{\circ}$ 2, p. 214-222, 2005.

T. Miyamoto, N. Rexha, «Determinants of three facets of customer trust: A marketing model of Japanese buyer-supplier relationship », Journal of Business Research, Vol. 57, N³, p. 312-319, 2004.

C. Moorman, G. Zaltman, R. Deshpande, « Relationships between providers and users of market research. The dynamics of trust within and between organisations ", Journal of Marketing Research, Vol. 29, $\mathrm{N}^{\circ} 3$, p. 314-28, 1992.

R. Morgan, S. Hunt, « The commitment-trust theory of relationship marketing », Journal of Marketing, Vol. 58, p. 20-38, 1994.

J.P. Neuville, «La stratégie de la confiance. Le partenariat observé depuis le fournisseur », Sociologie du Travail, Vol. 3, p. 297-319, 1997.

B. Nooteboom, H. Berger, N.G. Noorderhaven, «Effects of Trust and Governance on Relational Risk », Academy of Management Journal, Vol. 40, $\mathrm{N}^{\circ}$ 2, p. 308-338, 1997.

R.W Palmatier, R. P. Dant, D. Grewal, K.R. Evans, «Factors Influencing the Effectiveness of Relationship Marketing: A Meta-Analysis», Journal of Marketing, Vol. 70, N4, p.136-153, 2006.

J. M. Payan, (2006), «Multiple levels of trust and dependence on supplier-distributor coordination: an empirical test», Marketing Management Journal, Vol. 16, $\mathrm{N}^{\circ}$ 1, p. 125-137, 2006.

D. Puthod, «Entre confiance et défiance, la vigilance au coeur de la gestion des alliances », Gestion 2000, $\mathrm{N}^{\circ} 2$, p. 111-129, mars-avril 1995.

M. Sako, «Does trust improve business performance? », in C. Lane and, R Backmann (Ed.), Trust in and between Organisations, Oxford University Press, Oxford, 1998.
M. Sako, Price, Quality and Trust. Interfirm Relations in Britain and Japan, Cambridge University Press, Cambridge, 1992.

C. Schumacher, «Trust. Asource of success in strategic alliances?», Schmalenbach Business Review, Vol. 58, $\mathrm{N}^{\circ} 3$, p. 259-278, 2006.

F. Selnes, «Antecedents and consequences of trust and satisfaction in buyer-seller relationships », European Journal of Marketing, Vol. 32, N³/4, p. 305-322, 1998.

J.A. Siguaw, T.L. Baker, P.M. Simpson, «Preliminary evidence on the composition of relational exchange and its outcomes: the distributor perspective », Journal of Business Research, Vol. 56, N 4, p. 311-322, 2003.

J.A. Siguaw, P.M. Simpson, T.L. Baker, «Effects of supplier market orientation on distributor market orientation and the channel relationship: the distributor perspective », Journal of Marketing, Vol. 62, $\mathrm{N}^{\circ} 3$, p. 99-111, 1998.

J.T. Simpson, D.T. Mayo, «Relationship Management: A call for fewer influence attempts?», Journal of Business Research, Vol. 39, №3, p.209-218, 1997.

J.B. Smith, D.W. Barclay, «The effects of organizational differences and trust on the effectiveness of selling partner relationships », Journal of Marketing, Vol. 61, $\mathrm{N}^{\circ}$ 1, p. 3-21, 1997.

C. Wiertz, K. de Ruyter, C. Keen, S. Streukens, «Cooperating for service excellence in multichannel service systems: an empirical assessment », Journal of Business Research, Vol. 57, N 4, p. 424-432, 2004.

O.E. Williamson, «Calculativeness, trust, and economic organization », The Journal of Law and Economics, Vol. 34, p. 453-502, 1993.

R. Woolthuis, B. Hillebrand, B. Nooteboom, « Trust, Contract and Relationship Development», Organization Studies, Vol. 26, N 6, p. 813-840, 2005.

A. Zaheer, B. McEvily, V. Perrone, « Does trust matter: Exploring the effects of interorganizational and interpersonal trust on performance », Organization Science, Vol. 9, №2, p. 141-159, 1998.

A. Zaheer, N. Venkatraman, «Relational gouvernance as an interorganizational strategy: ann empirical test of the role of trust in economic exchange», Strategic Management Journal, Vol. 16, №5, p.373-392, 1995.

G.L. Zucker, «Production of Trust: Institutional Sources of Economic Structure, 1840-1920», in B.M. Staw et L.L. Cumings (eds), Research in Organization Behavior, Vol. 8, JAI Press, Greenwich, p. 53-111, 1986. 